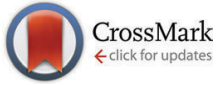

Cite this: Chem. Commun., 2016, 52,4373

Received 8th October 2015,

Accepted 18th February 2016

DOI: $10.1039 /$ c5cc08369a

www.rsc.org/chemcomm

\section{Synthesis, self-assembly, and photocrosslinking of fullerene-polyglycerol amphiphiles as nanocarriers with controlled transport properties $\dagger$}

\author{
levgen Donskyi, ${ }^{a}$ Katharina Achazi, ${ }^{a}$ Virginia Wycisk, ${ }^{a}$ Christoph Böttcher $^{\mathrm{b}}$ and \\ Mohsen Adeli*ac
}

In this work, we report a new, simple, gram-scale method for synthesizing water-soluble fullerene-polyglycerol amphiphiles (FPAs) that self-assemble into partially and fully crosslinked nanoclusters with the ability to controllably transport hydrophobic and hydrophilic agents.

Fullerenes belong to the firstborn class of modern carbon-based nanomaterials with well-defined structures and unique properties. ${ }^{1}$ Since they are hydrophobic compounds, their hybridization with the hydrophilic objects result in amphiphilic materials. Due to their special physicochemical properties, fullerene-based amphiphiles can be used to create nanoscale supramolecular structures. ${ }^{2}$ Conjugation of hydrophilic entities onto the surface of hydrophobic fullerenes is one way to prepare fullerene based amphiphiles. ${ }^{3,4}$ Alternatively, they can be synthesized by attaching fullerene salts to hydrophobic segments. ${ }^{5}$ One of the most relevant properties of the amphiphilic materials is that the fullerene-based amphiphiles can self-assemble into different types of nanostructures, depending on such physicochemical factors ${ }^{6}$ as concentration, polarity of the medium, and the chemical structure of the molecule conjugated to the surface of the fullerene. ${ }^{7}$ The last factor not only affects the structure of self-assemblies of fullerene-based amphiphiles and their physiochemical properties, but also dramatically dominates their biological behavior. For example, conjugation of fluorous chains onto the surface of fullerenes results in the formation of spherical vesicles in which nonpolar fluorous chains are exposed to an aqueous environment, ${ }^{8}$ while dodeca-dendronized fullerenes self-assemble into a disc-shaped structure where the fullerenes are located in the center. ${ }^{9}$ Hydrophilic macromolecules conjugated to fullerenes, on the other hand, improve their biological properties for biomedical applications. ${ }^{10,11}$ Furthermore, self-assemblies

\footnotetext{
${ }^{a}$ Institut für Chemie und Biochemie Organische Chemie, Freie Universität Berlin, Takustr. 3, 14195 Berlin, Germany.E-mail: aadeli@fu-berlin.de

${ }^{b}$ Forschungszentrum für Elektronenmikroskopie and Core Facility BioSupraMol, Institut für Chemie und Biochemie, Freie Universität Berlin, Berlin, Germany ${ }^{c}$ Department of Chemistry, Faculty of Science, Lorestan University, Khorram Abad, Iran.E-mail:mohadeli@yahoo.com

$\dagger$ Electronic supplementary information (ESI) available: Full experimental data and characterizations. See DOI: 10.1039/c5cc08369a
}

of fullerene-based amphiphiles can be fixed in the form of stable nanostructures by irradiation with UV light. ${ }^{12}$ This strategy is a promising approach to produce nanocarriers with controlled permeability and transport properties.

However, the critical issues for obtaining stable fullerene-based nanostructures with extraordinary transport properties are the type of hydrophilic polymer and a straightforward method to conjugate this polymer to the surface of fullerene. Photocrosslinking the assemblies of fullerene-based amphiphiles should not affect the other blocks, just the fullerene segments. Therefore, polymers without any significant absorption in the UV region are suitable candidates in this case.

Polyglycerol has been used as a highly water soluble, multifunctional, and biocompatible compound to prepare a wide range of nanostructures for promising nonmedical tools. ${ }^{13-15}$ Conjugation of polyglycerol to carbon-based nanomaterials not only enhances their water solubility and multi-functionality but also improves their biocompatibility. ${ }^{16,17}$ Moreover, it is almost transparent in the UV-vis region, which allows photocrosslinking of the fullerene-polyglycerol assemblies and production of nanocapsules in the aqueous solutions. Based on these envisioned outcomes, polyglycerol is a suitable candidate for producing fullerene-based amphiphiles that are able to create nanostructures with the new transport properties through molecular self-assembly and photocrosslinking in the aqueous solutions.

In this work, FPAs were synthesized on the gram scale using a one-pot "grafting from" method. The easy production of such amphiphiles is a big advantage for their practical application. Photocrosslinking the oligomeric assemblies of FPAs in aqueous solution resulted in nanoparticles with unusual transport properties. In addition, confocal microscopy showed that photocrosslinked assemblies are able to hold encapsulated dyes in a physiological medium for a long time without significant leakage.

Nucleophilic reaction between potassium tert-butoxide and fullerene led to tert-butoxy functionalized fullerenes with anionic centers that were able to initiate the anionic ring-opening polymerization of glycidol (Scheme 1). 


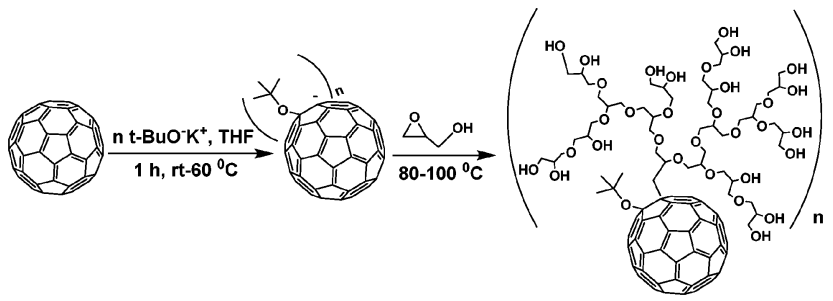

Scheme 1 Ring-opening polymerization of glycidol with a fullerene derivative that had anionic centers on its surface resulted in the formation of FPAs. The number of branches and degree of polymerization depended on the number of anionic centers and glycidol feed ratio, respectively.

The yield of reactions was high and in the range of $80-90 \%$. For example, the mass of the purified product of the reaction that was started using $4.4 \mathrm{~g}$ of glycidol and $0.3 \mathrm{~g}$ of fullerene was $4 \mathrm{~g}$, which confirms the gram-scale production of the FPAs using this approach.

These new FPAs were fully characterized using different spectroscopic and microscopic methods (see ESI $\dagger$ ). The number and molecular weight of polyglycerol branches, conjugated to the surface of fullerene, depended on the potassium tert-butoxide/ fullerene and glycidol/fullerene ratios, respectively (see Table S1 and Fig. S3, ESI $\dagger$ ). The number of polyglycerol branches attached to the fullerene segment was calculated using tert-butoxide/ polyglycerol peak area ratios in the ${ }^{1} \mathrm{H}$ NMR spectra (Table S2, ESI $\dagger$ ). Keeping the glycidol/fullerene ratio constant, increase in the potassium tert-butoxide/fullerene ratio resulted in a higher number of polyglycerol branches attached to fullerene. When these parameters were fixed in a ratio of $0.08 \mathrm{~mol} / 0.4 \mathrm{mmol}$ and $0.8 \mathrm{mmol} / 0.4 \mathrm{mmol}$, respectively, the produced FPAs were easily soluble in PBS, but had a high tendency to form aggregates. The critical aggregation concentration (CAC) of FPAs was measured at $14 \mu \mathrm{M}$ using the DLS method. ${ }^{18}$ Above CAC $(120 \mu \mathrm{M})$, they formed oligomeric assemblies with a hydrodynamic diameter of $20 \mathrm{~nm}$ (Fig. 1 and Fig. S7a, ESI $\dagger$ ).

In order to verify the morphology and size of the oligomeric assemblies, we performed cryo-TEM of the FPAs in PBS above the CAC. Fig. S8a (ESI $\dagger$ ) shows a monodisperse distribution of spherical particles with a mean diameter of $6 \mathrm{~nm}$ thought to correspond to the oligomeric assemblies (3-4) of FPAs.

Transport capacity (TC) and transport efficiency (TE) of fullerene-polyglycerol oligomeric assemblies for hydrophobic dyes, such as $\mathrm{Cy} 5-\mathrm{CH}_{3}$, were much higher than those for the hydrophilic dyes, such as Cy5-COOH (Table S3, ESI $\dagger$ ).

Based on cryo-TEM images, FPAs were thought to form oligomeric micelle-like assemblies, probably via hydrophobic interactions of the unfunctionalized fullerene domains, whereby a central hydrophobic core could encapsulate hydrophobic guest molecules (Fig. 2a). Therefore, they were able to transport Cy5- $\mathrm{CH}_{3}$ (Fig. 2b), whereas little transport effect was observed for Cy5-COOH (Fig. 2b).

However, many therapeutic agents are hydrophilic compounds and should be encapsulated and transported by the appropriate nanocarriers in order to improve their efficiency. ${ }^{19}$ Due to the dynamic nature of carriers that are assembled via weak noncovalent

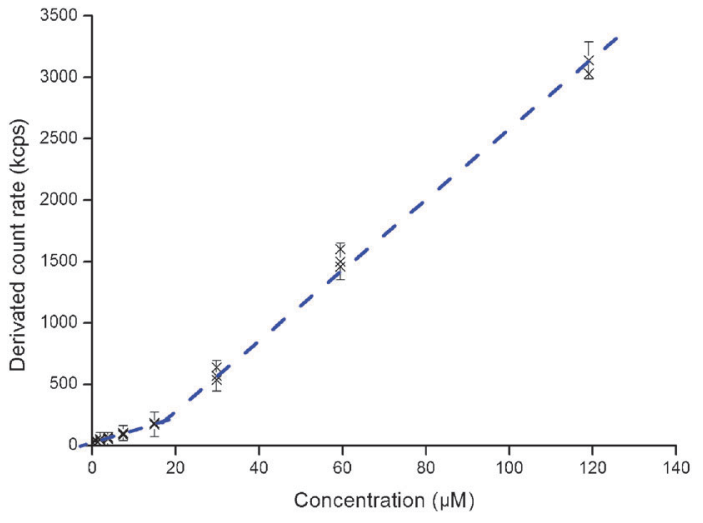

Fig. 1 Scattered intensity (kcps) as a function of FPAs concentration $(\mu \mathrm{M})$.

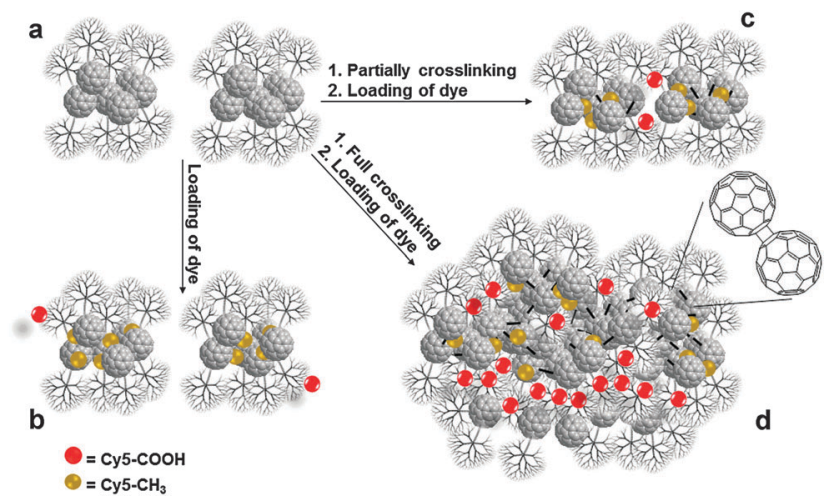

Fig. 2 Schematic representation of FPAs assemblies in different forms and their interactions with the guest molecules. (a) Oligomeric FPA assemblies, (b) hydrophobic interactions between $\mathrm{Cy}_{5}-\mathrm{CH}_{3}$ and FPAs, (c) and (d) partial and full crosslinking of oligomeric assemblies to produce bigger assemblies, respectively.

interactions, they do not provide sufficient stability under physiological conditions. Therefore, premature drug release and nonspecific biodistribution diminish their efficiency. Partial crosslinking of those nanocarriers, however, increases their stability and results in improved transport capacities and also controls the release of the encapsulated therapeutic agents. ${ }^{20,21}$

It has been proven that photocrosslinking of fullerene nanoparticles diminishes their permeability. ${ }^{12}$ Therefore, FPAs $(120 \mu \mathrm{M})$ in PBS were irradiated by a UV USHIO super high mercury lamp (USH 102d, $100 \mathrm{~W}$ ) to obtain partially crosslinked fullerene-polyglycerol assemblies (PCAs) with improved encapsulation properties.

The intensity of the $\lambda_{\max }$, which is characteristic for FPAs in the UV spectra and peaked at $280 \mathrm{~nm}$, was almost reduced by half after $3 \mathrm{~h}$ of irradiation in PBS, which evidences at least partial photocrosslinking of the fullerene moieties to form aggregates (Fig. 3). Longer UV irradiation furthered the crosslinking process but did not change the UV absorption of the maximum crosslinked (FCAs).

Since the brown color of the neat FPAs solution is due to the $\pi$-system of fullerenes, the conversion of the color to yellow and light yellow over the irradiation time made the gradual crosslinking of the fullerene segments visibly apparent (Fig. 3b and c). 


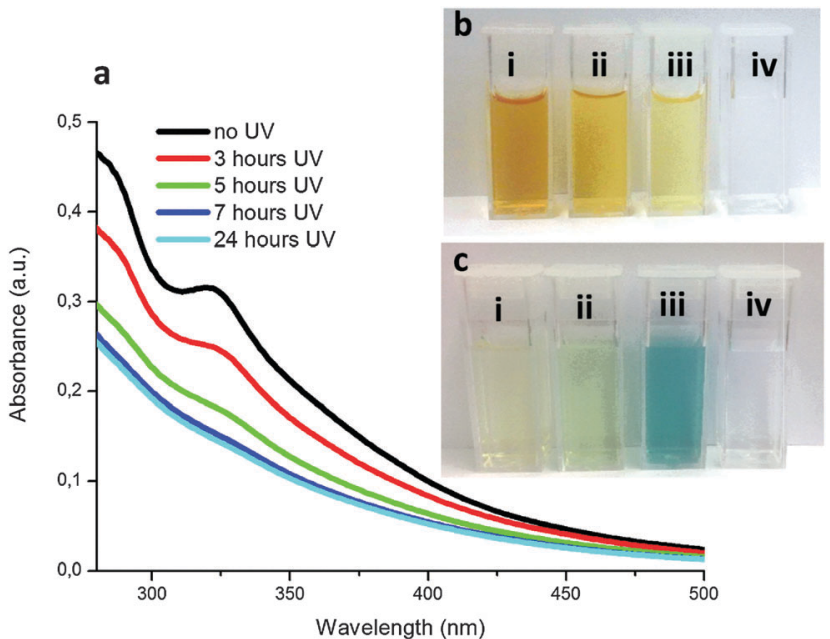

Fig. 3 (a) UV spectra of a $2 \mathrm{mg} \mathrm{ml}^{-1}$ PBS solution of FPAs irradiated at different times by a UV lamp at $100 \mathrm{~W}$. (b) Changes in the color of the $2 \mathrm{mg} \mathrm{ml}^{-1}$ PBS solution of FPAs from yellow to light yellow upon (i) $0 \mathrm{~h}$, (ii) $3 \mathrm{~h}$, (iii) $24 \mathrm{~h}$ UV irradiation, and (iv) the $2 \mathrm{mg} \mathrm{ml}^{-1} \mathrm{PBS}$ solution of polyglycerol (PG) as a blank indicates a progressive degree of crosslinking. (c) Photo of $1 \mathrm{mg} \mathrm{ml}^{-1}$ PBS solutions of encapsulated $\mathrm{Cy} 5-\mathrm{COOH}$ by (i) noncrosslinked assemblies (NCAs), (ii) PCAs, (iii) FCAs, and (iv) PG as a blank.

Cryo-TEM images show that photocrosslinking the oligomeric assembly predominantly generated larger spherical assemblies with diameters in the range from 100 up to $500 \mathrm{~nm}$ (Fig. 4a, b and Fig. S8b, c, ESI $\dagger$ ). The morphology of assemblies above $100 \mathrm{~nm}$ can deviate from the spherical geometry in the images due to a flattening within the embedding vitrified solvent layer, which was in the 100-200 $\mathrm{nm}$ range. Moreover, the magnified inset from Fig. $4 \mathrm{~b}$ shows a stereo image $\left(8^{\circ}\right.$-tilt) to prove the photolinkage effect of FCAs (here in a linear fashion). Photocrosslinking of the aggregates was also evaluated by DLS measurements (see the ESI $\dagger$ for more details).

Interestingly, crosslinking of the fullerene-polyglycerol assemblies by UV irradiation improved their encapsulation properties towards Cy5-COOH, but partially diminished their TC and TE for Cy5-CH $\mathrm{CH}_{3}$ (Fig. 5 and Fig. S9, S10, Table S3, ESI $\dagger$ ). Obviously, after covalent crosslinking of the fullerene segments upon a [2+2] cycloaddition reaction, the hydrophobic part of the assemblies was less accessible to the hydrophobic dye. Therefore, the TC and TE for $\mathrm{Cy} 5-\mathrm{CH}_{3}$ changed inversely versus the photocrosslinking time (Fig. 2c). Decreased permeability of FCAs upon irradiation reduced diffusion or release of the hydrophilic dye from the hydrophilic environment and therefore improved their TC and TE for Cy5-COOH (Fig. 2d).

However, hydrophobic and hydrophilic dyes were strongly trapped by FCAs and dye leakage from the nanoparticles in the physiological medium was much lower compared to their noncrosslinked analogs, as was proven by in vitro release experiments and cellular uptake study of $\mathrm{Cy} 5-\mathrm{CH}_{3}$ encapsulated by noncrosslinked assemblies (NCAs) and FCAs, respectively (Fig. S11, $\mathrm{ESI} \dagger)$. These carriers were loaded with almost the same amount of dye $\left(2 \times 10^{-4} \mu \mathrm{M}\right)$ and they were incubated with A549 cells with concentration $(10 \mu \mathrm{M})$ lower than the CAC of FPAs. Since NCAs were not stable under these conditions, $\mathrm{Cy} 5-\mathrm{CH}_{3}$ did not

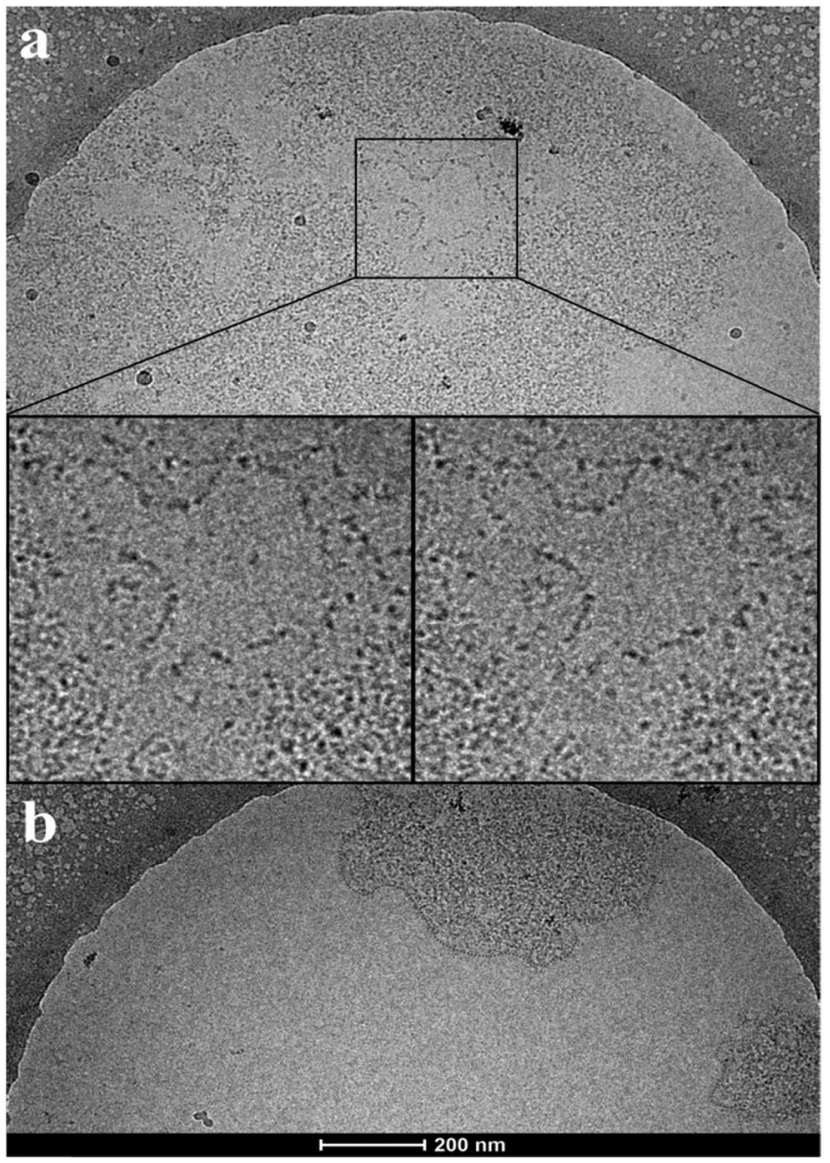

Fig. 4 Cryo-TEM images of FPAs in PBS after $7 \mathrm{~h}$ UV irradiation. Inset shows a stereo image of FCAs.

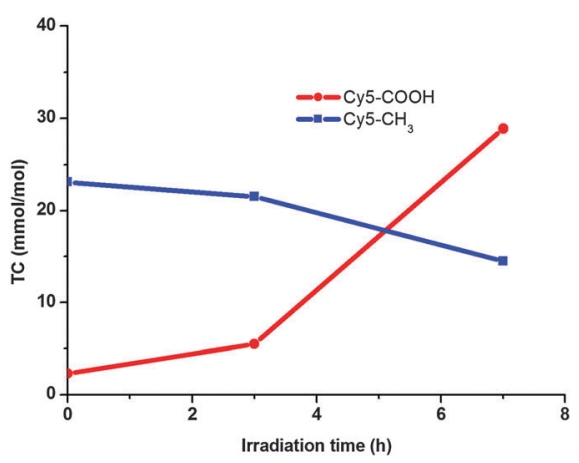

Fig. 5 Transport capacity of fullerene-polyglycerol assemblies for Cy5$\mathrm{COOH}$ and $\mathrm{Cy} 5-\mathrm{CH}_{3}$ versus irradiation time.

encapsulate and showed a similar uptake to the free dye (Fig. $6 \mathrm{~b}$ and c). Due to the covalent bonds, however, FPAs were stable even below the CAC and they were able to entrap the dye. Since they were larger clusters, as proven by cryo-TEM and DLS, their cellular uptake was supposed to be slow. Therefore, the uptake of encapsulated dye was likewise slow. Fig. $6 \mathrm{~d}$ shows the low fluorescence intensity of the encapsulated dye in comparison to the free dye or NCAs. Furthermore, cellular uptake studies after $24 \mathrm{~h}$ incubation time did not show any significant leakage of the dye encapsulated by FPAs, which confirmed the 


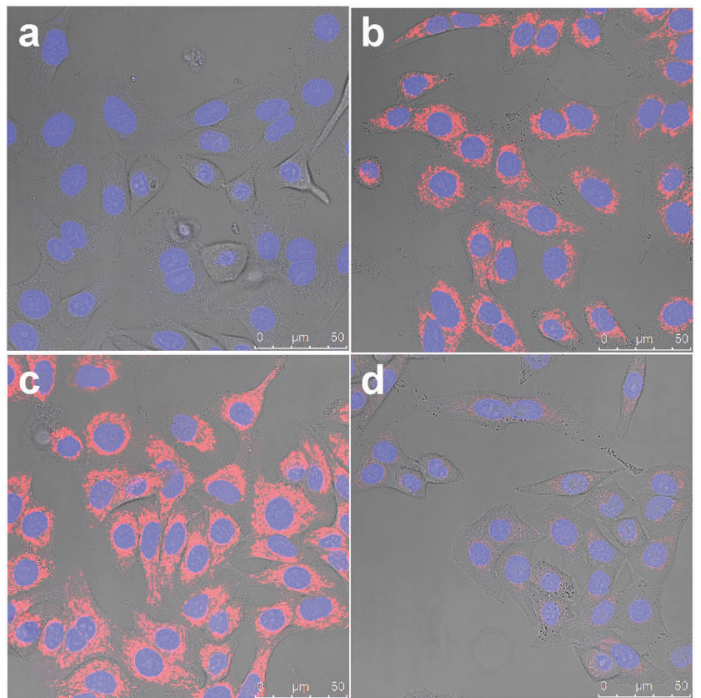

Fig. 6 Confocal microscopy images of the A549 cells. (a) Untreated (control), (b) treated with $2 \times 10^{-4} \mu \mathrm{M}$ of $\mathrm{Cy} 5-\mathrm{CH}_{3}$ dye, (c) and (d) $10 \mu \mathrm{M}$ of NCAs and FCAs loaded with $\mathrm{Cy} 5-\mathrm{CH}_{3}$, respectively, after 4 hours.

caging of $\mathrm{Cy} 5-\mathrm{CH}_{3}$ by this class of nanocarriers (Fig. S14, ESI $\dagger$ ). These results were further proven by flow cytometry experiments. A549 cells were incubated with FPAs and NCAs loaded with the same dye for $24 \mathrm{~h}$. Lower fluorescence intensity in the case of FPAs confirmed the low permeability of this nanocarrier and the slow release of the encapsulated dye (Fig. S15, ESI $\dagger$ ).

In summary, fullerenes can be used as a macroinitiator for anionic polymerization of glycidol, which leads to fullerenepolyglycerol amphiphiles. Size and transport properties of those amphiphiles in aqueous solution can be controlled via photocrosslinking of their hydrophobic fullerene segments.

The SFB 765 collaborative research center and the core facility BioSupraMol are gratefully acknowledged for financial support. Also, the authors would like to thank Dr Pamela Winchester for language polishing the manuscript.

\section{Notes and references}

1 A. Hirsch and M. Brettreich, Fullerenes: chemistry and reactions, Wiley-VCH, Weinheim, 2005.

2 R. Partha, M. Lackey, A. Hirsch, S. W. Casscells and J. L. Conyers, J. Nanobiotechnol., 2007, 5, 6, DOI: 10.1186/1477-3155-5-6.

3 A. Kahnt, D. M. Guldi, M. Brettreich, U. Hartnagelb and A. Hirsch, J. Mater. Chem., 2010, 20, 83.

4 N. Martín, F. Giacalone and J.-F. Nierengarten, Fullerene Polymers: Synthesis, Properties and Applications, Wiley-VCH, Weinheim, 2009.

5 T. Homma, K. Harano, H. Isobe and E. Nakamura, J. Am. Chem. Soc., 2011, 133, 6364.

6 A. P. J. Schenning and S. J. George, Nat. Chem., 2014, 6, 658.

7 Z. Lin, P. Lu, C.-H. Hsu, K. Yue, X.-H. Dong, H. Liu, K. Guo, C. Wesdemiotis, W.-B. Zhang, X. Yu and S. Z. D. Cheng, Chem. - Eur. J., 2014, 20, 11630.

8 T. Homma, K. Harano, H. Isobe and E. Nakamura, Angew. Chem., Int. Ed., 2010, 49, 1665.

9 S. Guerra, J. Iehl, M. Holler, M. Peterca, D. A. Wilson, B. E. Partridge, S. Zhang, R. Deschenaux, J.-F. Nierengarten and V. Percec, Chem. Sci., 2015, 6, 3393.

10 M. Lidija, T. Roumiana, M. Jelena, M. Mari, B. Kyoko, T. Marija and J. Branislava, Adv. Mater. Res., 2013, 633, 224.

11 R. Partha and J. L. Conyers, Int. J. Nanomed., 2009, 4, 261.

12 K. Harano, A. Narita and E. Nakamura, Chem. Lett., 2013, 42, 1176.

13 A. Sunder, R. Mülhaupt, R. Haag and H. Frey, Adv. Mater., 2000, 12, 235.

14 I. N. Kurniasih, J. Keilitz and R. Haag, Chem. Soc. Rev., 2015, 44, 4145.

15 K. Pant, D. Gröger, R. Bergmann, J. Pietzsch, J. Steinbach, B. Graham, L. Spiccia, F. Berthon, B. Czarny, L. Devel, V. Dive, H. Stephan and R. Haag, Bioconjugate Chem., 2015, 26, 906.

16 R. Soleyman and M. Adeli, Polym. Chem., 2015, 6, 10.

17 M. Adeli, R. Soleyman, Z. Beiranvan and F. Madani, Chem. Soc. Rev., 2013, 42, 5231.

18 Y. Skhiri, P. Gruner, B. Semin, Q. Brosseau, D. Pekin, L. Mazutis, V. Goust, F. Kleinschmidt, A. El Harrak, J. Hutchison, E. Mayot, J. Bartolo, A. Griffiths, V. Taly and J. Baret, Soft Matter, 2012, 8, 10618.

19 S. Vrignaud, J.-P. Benoit and P. Saulnier, Biomaterials, 2011, 32, 8593.

20 Y. Shao, W. Huang, C. Shi, S. T. Atkinson and J. Luo, Ther. Delivery, 2012, 3, 1409.

21 L. Yan, L. Yang, H. He, X. Hu, Z. Xie, Y. Huang and X. Jing, Polym. Chem., 2012, 3, 1300. 